\title{
EVIDENCE FOR ELECTRON EXCITATION OF TYPE III RADIO BURST EMISSION
}

\author{
H. ALVAREZ and F. HADDOCK \\ Radio Astronomy Observatory, University of Michigan, Ann Arbor, Mich., U.S.A. \\ and \\ R. P. LIN \\ Space Sciences Laboratory, University of California, Berkeley, Calif., U.S.A.
}

(Received 28 April, 1972)

\begin{abstract}
Type III radio bursts observed at kilometric wavelengths ( $\lesssim 0.35 \mathrm{MHz})$ by the OGO-5 spacecraft are compared with $>45 \mathrm{keV}$ solar electron events observed near $1 \mathrm{AU}$ by the IMP-5 and Explorer 35 spacecraft for the period March 1968-November 1969.

Fifty-six distinct type III bursts extending to $\lesssim 0.35 \mathrm{MHz}\left(\gtrsim 50 R_{\odot}\right.$ equivalent height above the photosphere) were observed above the threshold of the OGO-5 detector; all but two were associated with solar flares. Twenty-six of the bursts were followed $\lesssim 40$ min later by $>45 \mathrm{keV}$ solar electron events observed at $1 \mathrm{AU}$. All of these 26 bursts were identified with flares located west of W09 solar longitude. Of the bursts not associated with electron events only three were identified with flares west of W09, 18 were located east of W09 and 7 occurred during times when electron events would be obscured by high background particle fluxes.

Thus almost all type III bursts from the western half of the solar disk observed by OGO-5 above a detection flux density threshold of the order of $10^{-13} \mathrm{Wm}^{-2} \mathrm{~Hz}^{-1}$ at $0.35 \mathrm{MHz}$ are followed by $>45 \mathrm{keV}$ electrons at $1 \mathrm{AU}$ with a maximum flux of $\gtrsim 10 \mathrm{~cm}^{-2} \mathrm{~s}^{-1} \mathrm{ster}^{-1}$. If particle propagation effects are taken into account it is possible to account for lack of electron events with the type III bursts from flares east of the central meridian. We conclude that streams of $\approx 10-100 \mathrm{keV}$ electrons are the exciting agent for type III bursts and that these same electrons escape into the interplanetary medium where they are observed at $1 \mathrm{AU}$. The total number of $>45 \mathrm{keV}$ electrons emitted in association with a strong kilometer wavelength type III burst is estimated to be $\geqslant 5 \times 10^{32}$.
\end{abstract}

\section{Introduction}

Of the various forms of radio burst emissions from the Sun, type III bursts are most likely to be indicative of the escape of energetic particles from the Sun. These radio bursts exhibit a rapid frequency drift $\left(\sim 80 \mathrm{MHz}^{-1} \mathrm{~s}\right.$ at $\left.100 \mathrm{MHz}\right)$ from high to low frequencies and have been shown to be due to disturbances propagating outward in the solar corona at speeds of $\sim c / 3$ (Wild et al., 1954; 1959). Radio observations have identified these bursts over a frequency range from $\sim 500 \mathrm{MHz}$ (Maxwell et al., 1960) to $0.05 \mathrm{MHz}$ (Haddock and Alvarez, 1970) corresponding to a height range of $\sim 20000$ $\mathrm{km}$ to $\sim 100-200 R_{\odot}$ above the photosphere. It is clear therefore that at least for the type III bursts extending to low frequencies the exciting agent escapes from the Sun. The nature of the exciting agent has been hypothesized to be particles, both proton (Wild et al., 1954; Friedman and Hamberger, 1969) and electron streams (Wild et al., 1959), and magnetohydrodynamic shock waves (Westfold, 1957; Zirin, 1966). The latter appears unlikely in view of the large coronal magnetic fields required $(\sim 100 \mathrm{G}$ at $3 \times 10^{5} \mathrm{~km}$ height). 
There are theoretical grounds for the choice of proton streams as the excitation agent (Smith, 1970). However, protons of the requisite energy $(\sim 50 \mathrm{MeV})$ are rarely observed to be emitted by the Sun, and when they are emitted, they are not necessarily accompanied by type III radio emission but rather by type IV and type II (Lin, 1970). Smith (1970) raises the possibility that the proton flux from a type III burst is too small to be observed above galactic cosmic ray background by current spacecraft experiments, due to the diffusive nature of the propagation of these particles in the interplanetary medium. However, protons responsible for type III bursts would have velocities near $c / 3$ and thus would contribute to the background galactic cosmic ray spectrum in a limited energy interval about $50 \mathrm{MeV}$. That portion of the cosmic ray spectrum has been studied extensively for solar source and modulation effects (Kinsey, 1970 ) and there is no evidence for a long term solar component above $\sim 20 \mathrm{MeV}$.

On the other hand, solar electrons of the $\sim c / 3$ velocity $(\sim 32 \mathrm{keV}$ energy) are frequently observed in the interplanetary medium, although not nearly as frequently as metric and decametric wavelength type III bursts. Furthermore, almost every solar electron event of $\sim 40 \mathrm{keV}$ is accompanied by such type III burst emission (Lin, 1970). The main objections to the electron stream hypothesis for type II burst generation are (1) the relatively small number of electron events compared to type III bursts and (2) the difficulty of providing a theoretical explanation for the stabilization of the electron streams.

Type III bursts do not always drift down to very low frequencies. On many occasions bursts observed at metric wavelengths are not observed at decametric wavelengths, and similarly many bursts are not observed at hectometric and kilometric wavelengths which are seen by ground based observations which extend to decametric wavelengths (Hartz, 1969). Occasionally the reverse is also true. The reasons for these cutoffs are not understood at the present time. One possibility is that the exciting agent is impeded or dispersed in its progress outward through the corona.

In order to bypass the problem of interpretation of these cutoffs we have tabulated only type III burst which extend to kilometric wavelengths (frequencies $\$ 0.350 \mathrm{MHz}$ at height $\gtrsim 50 R_{\odot}$ ) and compared them with $>45 \mathrm{keV}$ electron events observed at $1 \mathrm{AU}$.

\section{Experiments}

The radio data is from the instrument that the University of Michigan has aboard the OGO-5 spacecraft (launched March 4, 1968). The electron data is provided by the University of California experiments aboard the Explorer 35 (AIMP-2) and Explorer 41 (IMP-5) spacecraft. The orbits of both spacecraft lie predominantly in the interplanetary medium. A detailed description of the Explorer 35 and OGO-5 instrumentation has been given elsewhere by Lin (1970) and MacRae (1968), respectively. Here it suffices to note that in Explorer 35, electron fluxes are unambiguously identified and are measured in two integral energy ranges, $>22 \mathrm{keV}$ and $>45 \mathrm{keV}$. IMP-5 provides comparable measurements in four integral electron energy ranges; $>18,>45,>780$ and $>120 \mathrm{keV}$. The events studied here are those with a maximum $45 \mathrm{keV}$ electron flux of 
$\gtrsim 10 \mathrm{~cm}^{-2} \mathrm{~s}^{-1} \mathrm{ster}^{-1}$. The OGO-5 radioastronomy receiver steps in $9.2 \mathrm{~s}$ through the following frequencies: $3.5,1.8,0.9,0.6,0.35,0.2,0.1$ and $0.05 \mathrm{MHz}$.

\section{Observations}

From the OGO-5 observations in March 1968 through November 1969 those type III bursts which clearly extend from $3.5 \mathrm{MHz}$ to $0.35 \mathrm{MHz}$ and down to $0.05 \mathrm{MHz}$ were tabulated. For each of these bursts the IMP and Explorer 35 data were examined for evidence of an electron event commencing within $\lesssim 40$ min after the type III burst onset time (observed at $1 \mathrm{AU}$ ) at $3.5 \mathrm{MHz}$.

To obtain the location of the type III emission region, each type III burst was identified with a solar flare under the criterion that the burst must occur within \pm 3 min of the period from flare start to flare maximum. We find that almost every one of the type III bursts observed by OGO-5 in the kilometric wavelength range was associated with a flare. This high correlation may be due to the high intensity of the type III burst necessary for detection by OGO-5. For four type III bursts, although no flare was found, an electron event was observed which had been attributed to an over-thelimb flare. A total of 26 of 56 distinct kilometric wavelength type III bursts located west of central meridian were followed by electrons observed at $1 \mathrm{AU}$ at an average of 25 min later.

Figure 1 shows an example of a type III burst followed by a solar electron event. There is a gap in the radio data between 0928 and 0932 UT and in the electron data between 0938 and $0948 \mathrm{UT}$. There was no flare associated with this event but the radio data give evidence that this burst was one in a sequence that originated in the McMath plage No. 10109 before and after it disappeared behind the west limb. We have estimated that the flare longitude was approximately between W120 and W130. Analysis of the radio event shown in Figure 1 (Haddock and Alvarez, 1971) indicated that the $0.1 \mathrm{MHz}$ burst was emitted as second harmonic at a heliocentric distance of $\gtrsim 120 R_{\odot}$ and arrived at the Earth not earlier than 0927 UT. If we assume that burst exciters or electron streams followed an Archimedes spiral that started $120^{\circ}$ west of the central meridian and passed through the Earth then the radio data arrival times at the Earth are consistent with exciters streams having a constant velocity with a value between $0.28 c$ and $0.38 c$. With these velocities the exciter particles were expected to arrive at the earth at 0939 and $0951 \mathrm{UT}$, respectively. The observations show that the $45 \mathrm{keV}$ solar electrons did not arrive earleir than 0936 UT but were already present at 0948 when the data gap was terminated. We conclude that the radio and particle data are consistent with a velocity $\sim 0.38 c$.

The locations of the type III emission are plotted in Figure 2. None of the observed electron events come from type III bursts located at longitudes east of W09; only three type III bursts out of 26 from flares located west of W09 did not give rise to electrons at $1 \mathrm{AU}$. The correlation to events originating in the western hemisphere of the Sun is consistent with the propagation characteristics of these electrons in the interplanetary medium. The Archimedean spiral field structure of the interplanetary field im- 
plies that field lines at the earth connect back to the vicinity of $\sim$ W50 solar longitude. Electrons do not propagate transverse to the interplanetary magnetic field lines (Lin et al., 1968) and the angular extent of the observed events from W09 to the west limb should be interpreted as due to an extended emission region (of $\gtrsim 50-60^{\circ}$ solar longitude) at or near the Sun.

We conclude that essentially every kilometric type III burst above the OGO-5 detection threshold and associated with a flare on the western hemisphere produces an electron event at $1 \mathrm{AU}$ with a maximum $>45 \mathrm{keV}$ flux of $\gtrsim 10 \mathrm{~cm}^{-2} \mathrm{~s}^{-1} \mathrm{ster}^{-1}$.

Graedel and Lanzerotti (1971) have questioned the association of solar electron events with type III bursts. Their result, however, does not consider the relevance of
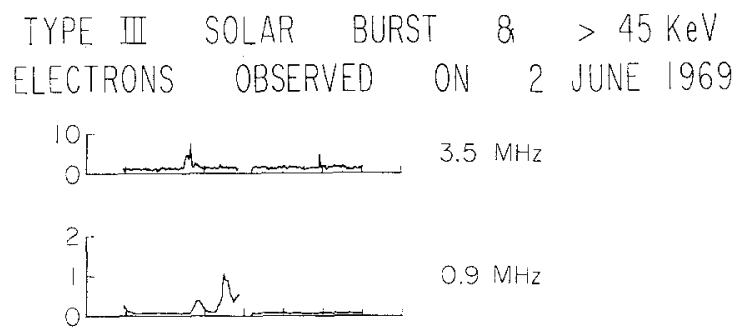

$0.9 \mathrm{MHz}$
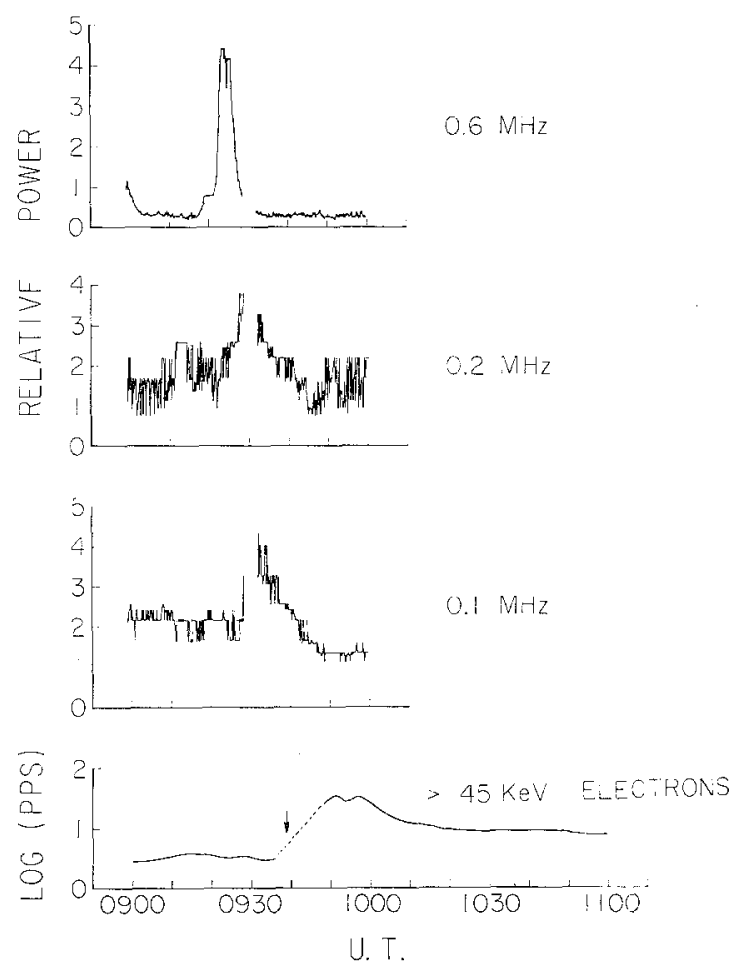

Fig. 1. Type III bursts observed at kilometric wavelengths by the OGO-5 spacecraft followed by $>45 \mathrm{keV}$ solar electrons observed by IMP-5 near 1 AU. The event occurred on 2 June 1969. The gaps in the radio data correspond to telemetry gaps. 


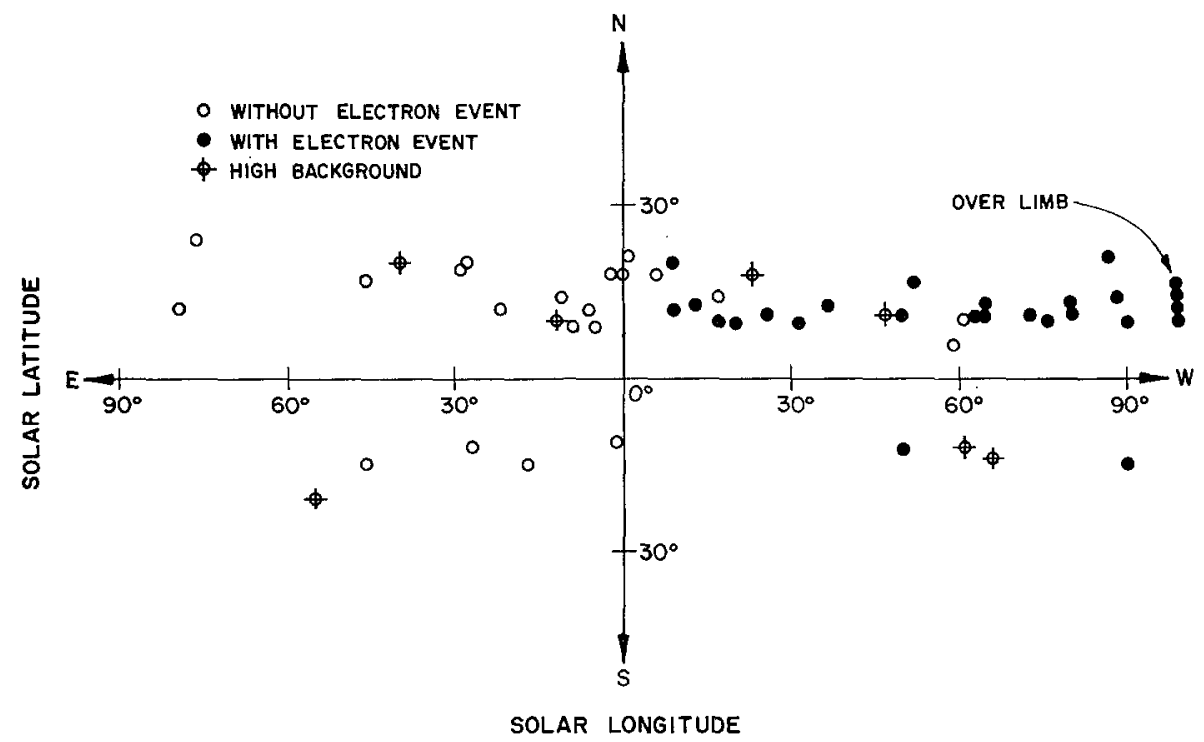

Fig. 2. Displayed here are the locations of the flares associated with each type III burst extending below $0.35 \mathrm{MHz}$. The bursts followed by solar electron events at $1 \mathrm{AU}$ are grouped in the west as expected from propagation considerations.

the associated flare position to the degree of solar electrons-radio burst correlations, as revealed in this study. It also does not consider the influence of the associated flare importance, as indicated by Sakurai (1971). Finally about $25 \%$ of the electron data they use $(>300 \mathrm{keV})$ corresponds to the elctron velocities $(\beta>0.77)$ out of range of most type III exciters. Further discussion of Gradel and Lanzerotti's paper is contained in Lin (1972), and Graedel and Lanzerotti (1972).

The maximum particle flux observed at $1 \mathrm{AU}$ may be used to derive the total number of electrons per burst emitted from the Sun, under some crude assumptions about the propagation of these particles on the interplanetary medium. Following the method of Lin and Hudson (1971), we find that the particle flux threshold of $\sim 10 \mathrm{~cm}^{-2} \mathrm{~s}^{-1}$ ster $^{-1}$ corresponds to $\geqslant 5 \times 10^{32}$ electrons released at the Sun.

\section{Summary}

Earlier studies of $\sim 40 \mathrm{keV}$ solar electron events have established that such events are are almost always accompanied by type III bursts at the time of particle injection into the planetary medium. Here we have presented evidence that essentially a one-to-one correspondence exists between kilometric wavelengths type III burst above a threshold of approximately $10^{-13} \mathrm{Wm}^{-2} \mathrm{~Hz}^{-1}$ and $>45 \mathrm{keV}$ electrons observed at $1 \mathrm{AU}$. We conclude that streams of $\sim 10-100 \mathrm{keV}$ electrons are the exciting agent for type III bursts, and that $\gtrsim 5 \times 10^{32}$ electrons with energy $>45 \mathrm{keV}$ are emitted in a strong type III burst. A problem which remains is to explain the cutoff of many type III bursts before they reach kilometric wavelengths. The present correlation suggests that such 
cutoffs may be due to the stopping or dispersion of the electron stream before it reaches $1 \mathrm{AU}$.

\section{Acknowledgements}

We acknowledge the contributions of the staff of the University of Michigan radio astronomy space program responsible for the success of the OGO-5 experiment. The OGO-5 research was funded by the NASA under contract NAS 5-9099 (University of Michigan). The Explorer 35 and IMP-5 research was supported in part by the NASA under contract NAS 5-9091 and grant NGL 05-003-017 (University of California).

\section{References}

Friedman, M. and Hamberger, S. M.: 1969, Solar Phys. 8, 398.

Graedel, T. E. and Lanzerotti, L. J.: 1971, J. Geophys. Res. 76, 6932.

Graedel, J. E. and Lanzerotti, L. J.: 1972, Reply to Lin (1972) submitted to J. Geophys. Res.

Haddock, F. T. and Alvarez, H.: 1970, Bulletin AAS 2, 196 (Abstract).

Haddock, F. T. and Alvarez, H.: 1971, Bulletin AAS 3, 6 (Abstract).

Hartz, T. R.: 1969, Planetary Space Sci. 17, 267.

Kinsey, J. H.: 1970, Phys. Rev. Letters 24, 246.

Lin, R. P.: 1970, Solar Phys. 12, 266.

Lin, R. P.: 1972, Comments on a brief report by T. E. Graedel and L. J. Lanzerotti, 'Interplanetary Particle Associations with Type UII Solar Bursts,' submitted to J. Geophys. Res.

Lin, R. P. and Hudson, H. S. : 1971, Solar Phys. 17, 412.

Lin, R. P., Kahler, S. W., and Roelof, E. C.: 1968, Solar Phys. 4, 338.

MacRae, B. D.: 1968, University of Michigan, Radio Astronomy Observatory Technical Report, September.

Maxwell, A., Howard, W. E., III, and Garmire, G.: 1960, J. Geophys. Res. 65, 3581.

Sakurai, K.: 1971, Solar Phys. 16, 125.

Smith, D. F.: 1970, Solar Phys. 15, 202.

Westfold, K. C.: 1957, Phil. Mag. 2, 1287.

Wild, J. P., Murray, J. D., and Rowe, W. C.: 1954, Australian J. Phys. 7, 439

Wild, J. P., Sheridan, K. V., and Neylan, A. A.: 1959, Australian J. Phys, 12, 369

Zirin, H.: 1966, Nature 212, 909. 